\title{
Criminologie
}

\section{Les défis de la réinsertion sociale}

Regard éclairé sur la réalité vécue par la clientèle en maison de transition

\section{Challenges to reintegration}

\author{
Barriers to reentry encountered by ex-convicts in halfway \\ houses
}

\section{Los desafíos de la reinserción social}

\section{Una mirada iluminada sobre la realidad vivida por la clientela en casa de transición}

\section{Marie-Ève Dubois et Frédéric Ouellet}

Volume 53, numéro 2, automne 2020

URI : https://id.erudit.org/iderudit/1074197ar

DOI : https://doi.org/10.7202/1074197ar

Aller au sommaire du numéro

Éditeur(s)

Les Presses de l’Université de Montréal

ISSN

0316-0041 (imprimé)

1492-1367 (numérique)

Découvrir la revue

Citer cet article

Dubois, M.-È. \& Ouellet, F. (2020). Les défis de la réinsertion sociale : regard éclairé sur la réalité vécue par la clientèle en maison de transition.

Criminologie, 53(2), 309-333. https://doi.org/10.7202/1074197ar

\section{Résumé de l'article}

La réinsertion sociale est un processus parsemé d'embûches au cours duquel un individu faisant face à la justice peut bénéficier d'interventions lui permettant d'être une personne autonome, intégrée à sa communauté et respectueuse des lois. Puisque l'état actuel des connaissances ne permet généralement pas d'aller au-delà de l'identification des difficultés qui surviennent dans ce processus, cette étude qualitative exploratoire a pour objectif d'examiner en profondeur les défis rencontrés par des hommes en maison de transition dans le cadre de leur processus de réinsertion sociale. L'analyse de contenu des entretiens $(n=16)$ a permis de distinguer six catégories de défis, soit le retour à la liberté, la situation financière et occupationnelle, le réseau social, le développement personnel, la stigmatisation et l'hébergement en maison de transition. Des particularités chez certains profils (durée de la sentence, délinquance sexuelle) ont été relevées. Les résultats sont discutés en regard des théories du désistement criminel. L'exercice souligne entre autres que certaines conditions légales et la stigmatisation des personnes judiciarisées créent des enjeux de contre-productivité. 


\title{
Les défis de la réinsertion sociale
}

\section{Regard éclairé sur la réalité vécue par la clientèle en maison de transition}

\author{
Marie-Ève Dubois ${ }^{1}$ \\ Doctorante \\ École de criminologie, Université de Montréal \\ marie-eve.dubois.1@umontreal.ca \\ Frédéric Ouellet \\ Professeur agrégé \\ École de criminologie, Université de Montréal \\ frederic.ouellet.1@umontreal.ca
}

RÉSUMÉ - La réinsertion sociale est un processus parsemé d'embûches au cours duquel un individu faisant face à la justice peut bénéficier d'interventions lui permettant d'être une personne autonome, intégrée à sa communauté et respectueuse des lois. Puisque l'état actuel des connaissances ne permet généralement pas d'aller au-delà de l'identification des difficultés qui surviennent dans ce processus, cette étude qualitative exploratoire a pour objectif d'examiner en profondeur les défis rencontrés par des hommes en maison de transition dans le cadre de leur processus de réinsertion sociale. L'analyse de contenu des entretiens $(n=16)$ a permis de distinguer six catégories de défis, soit le retour à la liberté, la situation financière et occupationnelle, le réseau social, le développement personnel, la stigmatisation et l'hébergement en maison de transition. Des particularités chez certains profils (durée de la sentence, délinquance sexuelle) ont été relevées. Les résultats sont discutés en regard des théories du désistement criminel. L'exercice souligne entre autres que certaines conditions légales et la stigmatisation des personnes judiciarisées créent des enjeux de contre-productivité.

MOTS CLÉS - Réinsertion sociale, interventions, libération conditionnelle, entretiens semi-dirigés, délinquance sexuelle.

1. École de criminologie, Université de Montréal, Pavillon Lionel-Groulx, C. P. 6128, succ. Centre-ville, Montréal (Québec), Canada, H3C 3J7.

Criminologie, vol. 53, $\mathrm{n}^{\circ} 2(2020)$ 


\section{Introduction}

La réinsertion sociale est l'un des objectifs derrière l'attribution d'une peine $^{2}$. En plus d'être une finalité légale, il s'agit du processus par lequel un individu reconnu coupable d'un crime peut bénéficier d'interventions visant le développement de capacités lui permettant d'être une personne autonome, respectueuse des lois et intégrée à sa communauté (Association des services de réhabilitation sociale du Québec [ASRSQ], 2014). La réinsertion sociale à la suite d'une période de détention est complexe; plusieurs études font ressortir des difficultés multiples associées au retour à la société (Petersilia, 2003). L'état des connaissances actuelles informe peu sur la manière dont les défis rencontrés dans ce processus nuisent au désistement criminel. À quelques exceptions près, la recherche dans ce champ ne permet pas de lier des caractéristiques individuelles aux difficultés rencontrées. Ces connaissances seraient toutefois pertinentes dans une perspective d'intervention en réinsertion sociale.

La présente étude souhaite explorer ces avenues et vise une compréhension approfondie des défis que pose la réinsertion sociale pour des individus ayant été incarcérés. Des entretiens semi-dirigés ont été réalisés auprès de résidents de maisons de transition.

\section{Les difficultés associées à la réinsertion sociale}

Il existe des barrières internes et externes au processus de réinsertion sociale et la nature des obstacles varie entre les individus (Brown, 2004). Les études antérieures ont soulevé des difficultés dans les sphères économique, professionnelle et sociale.

Visher, LaVigne et Travis (2004) ont montré qu'une majorité d'exdétenus ont des dettes importantes des mois après leur libération. Plusieurs individus étaient dans des situations économiques difficiles avant leur contact avec la justice et la judiciarisation a réduit leur accès aux services sociaux, à du financement, à des assurances ou à une marge de crédit (Brown, 2004). La précarité financière lors de la réinsertion sociale peut engendrer des difficultés comme l'incapacité à subvenir aux besoins de base (nourriture, hébergement, vêtements,

2. Cet objectif est prévu à l'article 718 du Code criminel, à l'article 3 de la Loi sur le système correctionnel et la mise en liberté sous condition et aux articles 2 et 3 de la Loi sur le système correctionnel du Québec. 
transport) (Harding, Wyse, Dobson et Morenoff, 2014; Morani, Wikoff, Linhorst et Bratton, 2011).

Parallèlement, la recherche d'emploi est un élément majeur d'une démarche de réinsertion sociale. La population judiciarisée est connue pour des déficits importants en matière de compétences, de qualifications, d'expériences professionnelles ou de motivation (Brown, 2004). Des problématiques de santé physique ou mentale et l'instabilité résidentielle constituent également des obstacles (Hardcastle et al., 2018). En plus de ces limites sur le plan individuel, la recherche d'emploi est complexifiée par une forte discrimination des employeurs à l'égard des personnes judiciarisées (Holzer, Raphael et Stoll, 2004). Le casier judiciaire stigmatise les condamnés, constituant une importante barrière à la réinsertion sociale (Tan, Chu et Tan, 2016). De même, le casier judiciaire réduit les possibilités d'accès à un appartement, un programme d'études et des relations sociales durables (Thornton, 2018).

L'établissement d'un réseau de soutien social pour les personnes judiciarisées est une difficulté bien documentée. La situation est d'autant plus problématique lorsque le milieu naturel valorise la délinquance (Brown, 2004) ou que le réseau social est composé de pairs criminalisés (Gendreau, Little et Goggin, 1996). Pour les individus ayant initialement un réseau plus sain, le maintien des relations prosociales avec la famille, la conjointe ou les enfants est sévèrement affecté par une incarcération (Comfort et al., 2018). La coupure, les conflits ou l'éloignement figurent parmi les éléments qui minent les relations préexistantes (Petersilia, 2003). Le développement de nouveaux liens sociaux avec les membres de la communauté peut être difficile (Brown, 2004). Par exemple, le dévoilement des antécédents judiciaires pose un frein aux individus dans les démarches de rencontres intimes (Harris, 2014). Moore, Stuewig et Tangney (2016) laissent entendre que tant la stigmatisation que le stigma anticipé nuisent à la réintégration communautaire.

Pour se réinsérer, les ex-détenus doivent parfois modifier leur attitude à l'égard de la vie conventionnelle et de certains comportements à risque (éthique de travail, impatience, stratégies d'adaptation inadéquates) (Brown, 2004). Par exemple, pour les individus aux prises avec une problématique de toxicomanie, la réinsertion sociale passera inévitablement par la régulation des habitudes de consommation. Cependant, des études relèvent que les contrevenants ne sont pas suffisamment informés par rapport aux services disponibles (Nhan, Bowen et Polzer, 
2017) et que l'accès à des programmes ou à des services communautaires est parfois limité (Seiter et Kadela, 2003).

Bien qu'il existe une littérature plutôt riche au sujet des difficultés associées à la réinsertion sociale, elle se limite généralement à l'identification de celles-ci. Des questionnements demeurent notamment quant aux interactions et aux relations possibles entre ces difficultés, au moment où celles-ci se présentent après la libération et à l'influence de certains facteurs sur le processus de réinsertion sociale. Par exemple, il est possible qu'une coupure importante avec la communauté créée par une longue incarcération donne lieu à des défis particuliers à la libération. Certaines études ont été effectuées auprès de clientèles spécifiques, dont les délinquants sexuels (voir Grossi, 2017). Une seule étude répertoriée procède à l'étude comparative avec un échantillon de la population générale de délinquants. Se penchant sur l'emploi et l'hébergement, les résultats obtenus par Rydberg (2018) laissent supposer que les délinquants sexuels rencontrent des difficultés spécifiques associées à leurs conditions de libération. Or, il y a un intérêt à explorer les spécificités associées à différents profils d'individus.

Enfin, la littérature suggère que dans leur processus de réinsertion sociale, les individus doivent relever plusieurs défis et ont de la difficulté à combler certains besoins essentiels à leur réussite. Cependant, les connaissances actuelles informent peu sur la manière dont ces défis ralentissent et nuisent au processus de désistement criminel.

\section{Étiquetage de la réhabilitation et transformation cognitive}

Les processus de réinsertion sociale et de désistement du crime ont des croisements conceptuels, dont une finalité commune; le succès du processus de réinsertion sociale se mesure entre autres par la cessation des comportements délinquants (Visher et al., 2004). En ce sens, il est utile d'avoir recours à des notions et à des théories du désistement pour examiner les défis rencontrés lors de la réinsertion sociale. Selon la théorie de l'étiquetage de la réhabilitation (Maruna, 2012), une personne judiciarisée se réhabilite par un processus de négociation de l'identité dans les interactions interpersonnelles. L'individu doit changer afin de se conformer aux normes de la société, mais la société doit également accepter sa réintégration pour qu'il y ait réhabilitation (Maruna, 2001). La conception de soi étant fonction de l'image reflétée 
par autrui (Maruna, Lebel, Naples et Mitchell, 2009), le processus de désistement ou de réinsertion sociale implique un étiquetage prosocial (Maruna, Lebel, Mitchell et Naples, 2004).

En continuité avec ces notions d'interactionnisme symbolique, Giordano, Cernkovich et Rudolph (2002) sont d'avis que le désistement criminel est rendu possible par une relation réciproque entre l'individu et son environnement. Dans leur formulation d'une théorie de la transformation cognitive, ils proposent que le désistement implique une ouverture au changement, une exposition à des grappins à changement, le développement d'une nouvelle identité et l'abandon du mode de vie associé à la délinquance. Les grappins à changement sont alors des opportunités dans l'environnement qui agissent à titre de catalyseur pour le changement identitaire (Giordano et al., 2002).

Ces théories révèlent plusieurs composantes nécessaires à la réussite du processus de désistement et de réinsertion sociale. Elles offrent alors un cadre théorique intéressant permettant d'observer la manière dont les obstacles rencontrés par les ex-détenus dans leur réinsertion sociale peuvent les freiner dans leur processus de désistement criminel.

\section{Présente étude}

Puisque le passage en maison de transition s'inscrit dans une logique de libération graduelle où les individus quittant un établissement de détention ont accès à des services visant à combler des besoins essentiels et à favoriser la progression des démarches de réinsertion sociale (ASRSQ 2014), il s'agit d'un environnement de choix pour étudier la réinsertion sociale. Sur la base des lacunes soulevées dans la littérature, la présente étude vise une compréhension approfondie des défis rencontrés par un échantillon de résidents de deux maisons de transition, libérés à la suite d'une période d'incarcération. Se basant sur des récits de vie narratifs, cette recherche s'intéresse à la perception des participants. Les centres d'hébergement visités accueillant une clientèle fédérale et provinciale $e^{3}$ ainsi que des individus ayant des antécédents de nature sexuelle, il est possible d'explorer les particularités de ces différents profils d'individus. Un regard éclairé sur le vécu d'ex-détenus

3. Ce qui distingue les clientèles fédérale et provinciale est la durée des sentences: en vertu de l'article 743.1 du Code criminel canadien, une condamnation à une détention de deux ans ou plus est purgée dans un établissement fédéral, alors que celles inférieures à deux ans sont purgées en établissements provinciaux. 
dans un contexte d'intervention en réinsertion sociale est susceptible d'avoir des implications pratiques pour les différents acteurs œuvrant dans ce domaine.

\section{Méthodologie}

\section{Provenance des données et participants}

Entre avril et août 2017, 16 entretiens semi-dirigés ont été réalisés auprès d'individus hébergés dans deux maisons de transition de la région de Montréal. Les participants ont été invités au moyen d'affiches de sollicitation et de la sollicitation directe par le personnel des centres d'hébergement. Ils ont été rencontrés en moyenne 3,9 mois après leur sortie de détention et avaient eu des peines variant de 6 mois à la perpétuité (moy. $=$ 53,2 mois, é.-t. $=77,9$ mois), s'agençant à toute une variété de délits commis. Aucun critère de sélection spécifique n'a été appliqué, le seul étant d'être hébergé dans les maisons de transition à la suite d'une période d'incarcération ${ }^{4}$. Puisque la sollicitation par le personnel a été la stratégie de recrutement principale, celui-ci a veillé à assurer une diversification des profils. L'échantillon est composé d'un nombre équivalent d'individus sous mandat fédéral (8) et provincial (8), et de neuf cas où le dernier crime commis était de nature sexuelle 5 .

Il importe de souligner que les objectifs, la méthode et les échéanciers de cette étude ont été déterminés en étroite collaboration avec la direction des maisons de transition selon ce qui était réaliste compte tenu du temps et des moyens à la disposition. La saturation empirique n'a pas été atteinte, mais très peu d'éléments nouveaux sont ressortis lors des derniers entretiens.

\section{Instruments et procédures}

L'entretien semi-directif est une méthode permettant un équilibre entre une structure imposée qui permet de cibler les éléments se rattachant à l'objet d'étude et une liberté offerte au participant dans l'étendue de ses réponses (Adams, 2015). Le déroulement des entrevues a été facilité

4. Certains individus y sont hébergés de manière exceptionnelle sans pour autant être en libération à la suite d'une incarcération.

5. Les individus ayant un antécédent de nature sexuelle pouvaient être judiciarisés au provincial (4) et au fédéral (5). 
par une grille d'entretiens composée des thèmes suivants: histoire de vie, trajectoire criminelle, expérience du système judiciaire, expérience de la réinsertion sociale, aide reçue/participation à des programmes. Les thèmes ont été abordés selon une logique chronologique permettant aussi une entrée graduelle dans les sujets plus délicats.

Les entretiens ont eu lieu dans les bureaux des maisons de transition et étaient d'une durée approximative de deux heures. Ils ont été enregistrés sur support audio, avec l'approbation des participants, afin d'être transcrits pour les analyses ${ }^{6}$.

\section{Profil des participants}

Tous les participants étaient des hommes d'origine canadienne, francophones, âgés de 24 et 68 ans au moment des entretiens (moy. $=44,2$, é.-t. $=12,8)$. La moitié n'était pas en couple lors de la collecte $(\mathrm{n}=8)$ alors que la majorité l'était lors de l'arrestation $(n=12)$. Le jour de l'entrevue, neuf hommes étaient sans emploi. Néanmoins, tous ont à un moment ou à un autre été à l'emploi de manière légitime dans leur vie. Ils avaient occupé des emplois dans des domaines variés tels que la construction, la vente, l'informatique, la restauration et la mécanique.

L'histoire de vie des hommes rencontrés est pour la majorité marquée par un parcours difficile. Plusieurs soulignent avoir connu des modèles parentaux inadéquats $(n=9)$, un parcours scolaire difficile $(n=11)$ et des problématiques de dépendances aux drogues, à l'alcool ou au jeu $(\mathrm{n}=11)$. Sept participants ont un historique de délinquance juvénile à gravité variable. À l'âge adulte, les participants ont eu des trajectoires criminelles pour la plupart diversifiées. Ceux-ci ont été impliqués dans la délinquance lucrative $(n=8)(p$. ex.: criminalité de marché ou contre la propriété), la délinquance violente $(\mathrm{n}=7)(\mathrm{p}$. ex. : voies de fait, vols qualifiés, homicides) et la délinquance sexuelle $(n=8)$ (p. ex. : production/distribution de pornographie juvénile, voyeurisme, agression sexuelle). Pour une partie de l'échantillon, la trajectoire criminelle se résume au délit pour lequel ils étaient en transition $(n=5)$ et c'est le cas principalement d'individus ayant commis un crime de nature sexuelle. Interrogée sur leur dernière période de détention, la majorité

6. Ce projet a été évalué puis accepté par le Comité d'éthique de la recherche en arts et sciences de l'Université de Montréal (CERAS-2016-17-294-D). Bien que la stratégie de recrutement soit un tri expertisé, les résidents ayant participé aux entretiens l'ont fait sur une base volontaire. 
TA B LEA U 1

Profil des participants

\begin{tabular}{|c|c|c|c|c|c|c|c|c|}
\hline Noms* & Âge & Dernière condamnation & $\begin{array}{l}\text { Dernière } \\
\text { sentence }\end{array}$ & $\begin{array}{c}\text { Temps depuis } \\
\text { la sortie }\end{array}$ & État matrimonial & $\begin{array}{c}\text { Nombre } \\
\text { d'enfants }\end{array}$ & En emploi? & Diplôme \\
\hline André & 44 & $\begin{array}{l}\text { Crimes violents et } \\
\text { contre la propriété }\end{array}$ & 18 mois & $21 / 2$ mois & Divorcé & 1 & oui & DES \\
\hline Bernard & 48 & Criminalité de marché & 10 mois & 3 mois & Célibataire & 1 & non & SEC2 \\
\hline Christian & 41 & Criminalité de marché & 14 mois & 5 sem. & Conjoint de fait & 2 & oui & $\begin{array}{l}\text { DES } \\
\text { DEP }\end{array}$ \\
\hline Daniel & 29 & Criminalité de marché & 9 mois & 2 mois & Conjoint de fait & 3 & non $^{\mathrm{a}}$ & SEC3 \\
\hline Émile & 54 & Crime sexuel & 20 mois & 3 mois & Marié & 1 & oui & $\begin{array}{l}\text { DES } \\
\text { DEP }\end{array}$ \\
\hline Félix & 47 & Crime sexuel & 6 mois & 1 mois & Marié, séparé & 2 & non & BAC \\
\hline Gérard & 24 & Crime sexuel & 15 mois & 6 mois & Marié & 2 & oui & DES \\
\hline Hugues & 42 & Crime sexuel & 1 an & 1 mois & Conjoint de fait & 1 & oui & SEC3 \\
\hline Julien & 60 & Crime violent & 12 ans & 5 mois & Veuf & 2 & non & DES \\
\hline Kévin & 28 & Crimes violents & ND & 10 mois & Célibataire (en couple) & 0 & oui & DES \\
\hline Léo & 35 & Crime sexuel & $31 / 2$ ans & 3 mois & Célibataire & 1 & oui & DES \\
\hline Marc & 53 & Criminalité de marché & 42 mois & 7 mois & Célibataire & 1 & non $^{a}$ & DES - DEP \\
\hline Nathan & 62 & Crime sexuel & Perpétuité & 9 mois & Veuf & 0 & non & $\mathrm{DEC}$ \\
\hline Olivier & 39 & Crime sexuel & 38 mois & 4 mois & Célibataire (en couple) & 1 & non & DES \\
\hline Pierre & 33 & Crime sexuel & 2 ans et 8 mois & 2 sem. & Marié & 4 & non & SEC3 \\
\hline Quentin & 68 & Crime sexuel & 8 ans & $41 / 2$ mois & Célibataire & 3 & non & DEP \\
\hline
\end{tabular}

* Des prénoms fictifs sont utilisés pour préserver la confidentialité des participants. a Aux études, payé par Emploi Québec.

DES: diplôme d'études secondaires; DEP: diplôme d'études professionnelles; DEC: diplôme d'études collégiales; BAC: baccalauréat. 
d'entre eux la considère traumatisante $(n=4)$, difficile $(n=3)$ ou vécue dans la peur $(\mathrm{n}=5)$. Ils ont parfois travaillé, étudié ou pris part à différents programmes pendant leur détention, ces opportunités étant toutefois moindres pour les participants provenant de centres de détention provinciale.

\section{Stratégie d'analyse}

Une analyse thématique de contenu du discours des participants a été effectuée à partir des transcriptions des entretiens. Comme suggéré par Trahan et Stewart (2013), une procédure en 6 étapes a été suivie. Après la prise de connaissance des données (1), une codification préliminaire (2) a été réalisée: l'objectif est de réduire la quantité de données afin qu'elles soient dans une forme plus simple (Roulston, 2014). Un codage de type in vivo - codes tirés directement du vocabulaire du participant - a été fait manuellement (Miles, Huberman et Saldaña, 2014). Des fiches résumées des codes préliminaires ont été produites pour chacun des participants. Par une analyse horizontale des données, des thèmes généraux ont été déterminés (3); les données issues des différentes entrevues ont été croisées afin de dresser la liste des thèmes récurrents, des modèles ou des différences (Miles et al., 2014). Ayant recours à la littérature, tout en conservant une ouverture aux éléments nouveaux (Roulston, 2014), le regroupement des codes a permis la constitution de classes de défis de la réinsertion sociale. C'est d'ailleurs à cette étape qu'une attention a été portée pour relever les particularités des différents profils d'individus. La vérification de l'exactitude des thèmes (4) a été faite conjointement par les coauteurs. Trahan et Stewart (2013) suggèrent d'observer la cohérence de chacun des thèmes généraux (homogénéité interne) et l'exclusivité de ceux-ci (hétérogénéité externe). Une fois la classification finalisée, les grands thèmes ont été définis (5) et l'information a été organisée de façon claire et concise (6) afin de les présenter.

\section{Résultats}

Les résultats présentés visent à déterminer et comprendre les défis que les participants ont affrontés, mais aussi à relever les spécificités relatives aux différents profils. L'analyse met en lumière six catégories de défis (Figure 1). 
FIG URE 1

Les défis associés à la réinsertion sociale

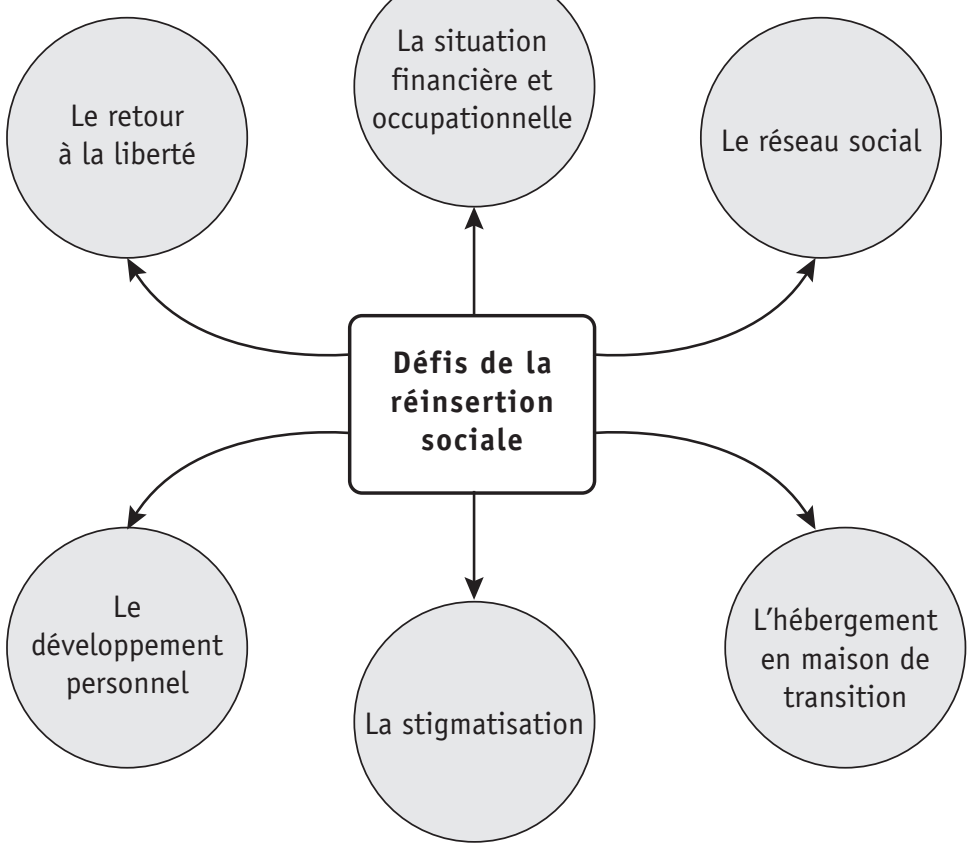

Le retour à la liberté

Des défis sont rencontrés dans la transition de la détention ferme à la liberté conditionnelle. À leur arrivée en maison de transition, huit participants $(8 / 16)$ avaient une multitude de démarches à entreprendre, dont effectuer des achats de base (p. ex.: produits d'hygiène, vêtements), faire la mise à jour ou obtenir différents documents légaux ou officiels (p. ex. : permis de conduire, carte d'assurance maladie, certificat de naissance, contraventions impayées, déclarations fiscales) et prendre divers rendez-vous médicaux. Les participants se disaient démunis tant en ce qui a trait aux moyens qu'aux connaissances requises pour effectuer ces tâches. Un individu a expliqué que le changement brusque de rythme entre la vie en détention et celle en liberté conditionnelle rend le retour en société d'autant plus anxiogène. La réintégration à la communauté implique de se réapproprier une routine quotidienne qui res- 
pecte les conditions légales, ce qui s'est avéré complexe dans certaines situations. Des participants ont mentionné éprouver de la difficulté à concilier la vie de famille, la vie en maison de transition, les programmes, le travail et toutes leurs autres responsabilités.

Parce que moi, je suis habitué à: je vais à l'école puis là, je relaxe dehors. Tu sais, veut veut pas, la prison, c'est au ralenti. Tu sais, on fait toujours la même chose. Mais ici, faut qu'on aille travailler, faut que je fasse ça, faut payer les bills, faut aller faire le marché... tu sais, la vie est super stressante debors comparée à là-bas. - Kevin

\section{La situation financière et occupationnelle}

L'argent était une préoccupation pour sept participants (7/16), celle-ci étant plus prévalente chez la clientèle provinciale (6/7). La difficulté à assumer divers paiements (comptes, carte d'autobus, permis de conduire, immatriculation) était une source d'anxiété. Bien que certains aient précisé avoir été dans une situation financière difficile avant les procédures judiciaires, plusieurs participants ont reconnu des facteurs contributifs à leurs difficultés précisément en lien avec leur statut de libéré conditionnel : l'inaccessibilité au chômage suivant une détention, les coûts associés à l'hébergement en maison de transition (loyer, multiples déplacements) et l'accès restreint à des services financiers ou d'assurances.

Je te dirais, pas mal tout le long de mon séjour ça a été une grosse préoccupation puisque, habiter ici coûte de l'argent, l'essence coûte de l'argent, la thérapie coûte de l'argent puis c'est pas des petits frais. - Gérard

Contribuant à ces difficultés, l'emploi a été jugé par un peu plus de la moitié de l'échantillon comme un défi de la réinsertion sociale (9/16). Selon ces participants, la recherche d'emploi avec un casier judiciaire constitue un défi de taille, et davantage avec un antécédent de nature sexuelle. La mention des antécédents judiciaires dans le processus d'embauche donnait lieu à des refus quasi systématiquement. Une grande proportion des individus ayant des antécédents de nature sexuelle avaient connu cette expérience. Outre la discrimination, les délinquants sexuels n'avaient accès qu'à un bassin d'emplois restreint en raison de leurs conditions généralement plus restrictives. Ces conditions empêchaient parfois le retour à leur emploi antérieur et forçaient une réorientation professionnelle. 
Je suis allé voir quand même des anciens employeurs puis ils ont dit: "Écoute, t'es dans la vente Émile.» Il dit: "On peut pas te mettre dessus, dessus le front dans un garage là, regarde on est chez [nom du concessionnaire], tu verrais-tu un moment donné: [nom du concessionnaire] eub... pédophile eub... tu sais. La publicité qu'on va avoir là. C'est pas personnel envers toi.» Mais il dit: «Il faut que tu comprennes la business.»-Émile

Si éviter de dévoiler le casier judiciaire dans le processus d'embauche limite aux premiers abords la discrimination, cela engendre des conséquences quant à la capacité à conserver l'emploi. Alors qu'il n'est pas légalement obligatoire de dévoiler ses antécédents judiciaires si la question n'est pas posée explicitement par un employeur, il arrive qu'il devienne au fait de cette information plus tard (p. ex. : article de journal). Quatre des personnes rencontrées (tous délinquants sexuels) ont perdu un emploi après la découverte de leurs antécédents par leurs employeurs. Étant conscients de ces risques, certains individus, à l'emploi au moment des entrevues, vivaient du stress relativement à leur secret en milieu de travail qui les mettait dans des situations inconfortables (p. ex.: prendre congé pour aller en thérapie). Finalement, pour deux individus ayant l'habitude d'obtenir un revenu par des moyens illégaux, l'emploi légitime était un défi, considérant l'écart important avec la charge de travail et les revenus antérieurs.

C'est sûr qu'elle (l'argent) est pas mal plus dure à gagner en ce moment, ça me fait chier de me lever à tous les matins à $6 \mathrm{~b}$ du matin pour aller travailler puis de faire 395 piasses dans une semaine quand je faisais... je pouvais faire ça par jour, là. - Daniel

Quelques participants faisaient un retour aux études, ce dernier étant imposé pour certains (réorientation professionnelle due aux conditions) et volontaire pour d'autres. Certains individus l'ont noté comme un défi dans leur réinsertion sociale relativement à deux facteurs. D'abord, l'âge était un enjeu dans la mesure où il serait plus complexe d'apprendre un métier passé la cinquantaine. Ensuite, obtenir l'autorisation d'être aux études était problématique pour deux individus ayant pour condition l'interdiction de se trouver en présence de personnes mineures. Il fallait alors des délais importants pour obtenir des exceptions à ces conditions. Un résident expliquait l'incohérence entre ses conditions de libération et son plan de sortie où l'éducation se trouve à la base du projet de réinsertion sociale: 
Alors maintenant, je peux plus aller à l'école. Parce que ceux qui vont à l'école ont de 16 à 18. Mais ils sont d'accord pour mon plan, mais à cause qu'ils m'ont mis ça, je peux plus aller à l'école. Mais "ab votre plan», si on voit le rapport, c'est écrit: «votre plan est... atteignable, il est mesurable et atteignable» [...]. Ils approuvent, mais ils me mettent des batons dans les roues. - Pierre

Le logement s'avérait une préoccupation pour six résidents $(6 / 16)$, presque tous des délinquants non sexuels (4/6), de la clientèle fédérale $(4 / 6)$ et majoritairement célibataire $(4 / 6)$. C'est en ayant en tête leur sortie, imminente pour certains, qu'ils ont abordé ces préoccupations. Outre la question des coûts, le défi était de déterminer où ils se logeraient à la sortie des centres d'hébergement. Pour trois participants de la clientèle fédérale prévoyant louer un appartement, des difficultés sous-jacentes au fait de vivre seul étaient anticipées: acheter le nécessaire pour meubler/équiper un logement et développer une routine.

Là, j'en ai un peu, de l'argent de ramassé, mais... moi ça me prend tout là, à partir du couteau là, jusqu'au frigidaire là, tu sais. Faut que je... Faut tout que je rachète, j'ai plus rien rien rien. - Julien

Ça va mal aussi ça se chercher un coloc[ataire]. «Hey by the way, je sors de prison, un supermaximum, une peine de 18 mois.»-André

\section{Le réseau social}

Plusieurs des défis rencontrés se trouvaient dans la sphère relationnelle. Un cumul des difficultés menait dans certains cas à l'isolement social. D'abord, les participants se sont heurtés à des défis dans leurs relations familiales (13/16). Cinq participants ont évoqué la nécessité de résoudre des conflits avec certains membres de la famille qui n'acceptaient pas les actes délictueux commis. La majorité (8/13) de ceux qui ont abordé ce thème avait commis un crime de nature sexuelle où la victime était un membre de la famille proche ou élargie, ce qui peut expliquer le fait que les familles ont pris leurs distances. Les conditions d'interdiction de contact avec les victimes, la famille des victimes ou bien les personnes mineures constituaient des barrières pour six individus, principalement pour maintenir le contact avec leurs enfants. Pour les délinquants sexuels dont la victime était mineure, trouver des activités familiales qui respectent les conditions était un casse-tête vu les conditions de l'article 161 du Code criminel qui limitent l'accès aux parcs, aux centres communautaires ou aux lieux où se trouvent généralement des personnes mineures. 
Un moment donné j'ai demandé: "Je peux-tu aller au Biodôme avec ma femme et mon garçon?» On me dit: "Non. Parce que ça fait partie de tes conditions.» Je suis comme, je me dis... je suis avec ma femme et mon garçon, qu'est-ce qui peut arriver? [...] Fait que, je comprends le fondement [des conditions], mais ça m'empêche de vivre de bons moments avec ma famille, ça m'empêche aussi de donner des expériences à mon garçon. - Gérard

Les participants ont mentionné des difficultés dans les relations conjugales. Pour ceux dont la relation de couple avait résisté à l'incarcération, la poursuite de cette relation était un défi. La résolution de conflits était souvent nécessaire pour ce type de relation aussi. Les conditions de l'article 161 avaient un impact sur les relations avec les conjointes, limitant l'éventail des activités de couple et l'impossibilité de se voir en présence des enfants. Pour six des huit célibataires de l'échantillon, le défi était plutôt de développer une relation conjugale (la plupart faisaient partie de la clientèle fédérale). Le dévoilement des antécédents judiciaires était une source d'anxiété pour plusieurs qui craignaient une mauvaise réaction de la partenaire. Si une relation demeurait possible après le dévoilement, l'enquête communautaire ${ }^{7}$ posait un frein aux participants qui avaient un malaise à imposer ce type de vérification aux nouvelles personnes rencontrées.

Aussitôt que je rencontre quelqu'un, que je parle à quelqu'un, c'est tout de suite une enquête communautaire et blablabla tu sais. Des fois, le monde sont pas intéressés à ce que tu ailles fouiller dans leur vie tu sais. C'est correct que tu sois leur ami, ils vont tout te dire, tu peux tout leur partager, mais tu sais, eux autres, ils sortent pas de prison là. - Marc

Ces barrières posaient également des limites sur le plan amical. En fait, que ce soit parce qu'ils étaient isolés avant la commission du délit ou parce qu'ils l'étaient devenus à la suite de la judiciarisation, plusieurs participants cherchaient à se recréer un réseau social $(7 / 16)$. Cherchant à développer de nouveaux liens, un sentiment d'infériorité par rapport aux autres contraignait l'approche de nouvelles personnes. Alors que la participation à des activités sociales aurait été le meilleur moyen de faire des rencontres, les participants se disaient restreints par leurs conditions légales. En plus de l'interdiction d'accès aux débits de boisson, les individus ayant commis un crime de nature sexuelle à l'égard d'un

7. Elle consiste en une rencontre avec un intervenant dans le but de vérifier que la personne est une influence positive et qu'elle est aux faits du délit commis et des conditions de libération. 
enfant se voyaient plus particulièrement affectés par les conditions de l'article 161. En fonction des lieux, ils étaient entre autres restreints dans le choix de certaines activités sportives ou culturelles.

J'ai quand même de la misère à dealer avec ça. Comme je te dis, je jouais au deck hockey puis... je joudis au baseball tu sais puis, ça se passe tout dans des centres communautaires ou bien des parcs. Fait que je peux pas y aller. Je voulais aller au gym. Là, sont en train de checker si je peux aller, parce qu'on avait le YMCA, il y a une piscine. Tu sais, oublie ça, je peux pas aller là. Je peux pas aller à la piscine là tu sais là. Fait que tu sais, il y a plusieurs affaires que je peux pas faire. Au début, je me disais, j'aurais commis un meurtre, je pense que j'aurais moins de conditions puis c'est vrai tu sais... c'était moins mal vu. - Léo

\section{Le développement personnel}

Plusieurs participants sortaient de détention avec des difficultés d'ordre personnel, dont des problématiques antécédentes à l'incarcération non résolues ou en cours de résolution (14/16). Étant donné qu'il s'agissait d'éléments ayant mené à la commission du délit, la résolution de ces problématiques se trouvait à la base même de l'idée de leur réinsertion sociale. Ainsi, trouver une solution à une problématique sexuelle $(5 / 16)$, de toxicomanie (4/16), de gestion des émotions/de l'estime de soi (7/16) ou en lien avec l'appât du gain $(2 / 16)$ comptait parmi les défis. Par ailleurs, une difficulté d'accès aux programmes ou aux services spécialisés a été relevée: accès réduit en raison des coûts des thérapies, listes et délai d'attente pour des thérapies spécialisées ou des services psychologiques. Cette situation était plus présente chez les délinquants sexuels.

Mais le gros problème, c'est avec mettons quand qu'ils t'imposent un programme comme avec le [programme pour délinquance sexuelle], eux autres, moi regarde ça fait depuis le mois de février, j'ai aucune idée quand est-ce que m’a rentrer là-dedans. Peut-être au mois de juin. C'est 64 semaines. Puis je suis obligé de rester ici parce que les... les commissaires ils ont marqué comme quoi que je devais être en maison de transition pendant que je prends mes programmes. Fait que je suis ici pendant 1 an et demi. Si j'avais décidé de faire mon deux tiers, [ça aurait été plus court]. [...] Là, dans tes conditions, tu dois faire une thérapie en psychologie avec une psychologue qui est complètement à part du [programme pour délinquance sexuelle]. Bien, je veux bien la faire la maudite thérapie. Là, j'ai attendu eub... on m'avait référé à une place. J'ai attendu un mois, téléphone, message, téléphone, message, j'avais fait pendant 1 mois et demi pour me faire dire: "T'es en maison de transition, on te prend pas.»-Émile 
Outre ces problématiques antécédentes à l'incarcération, la gestion du stress était un défi à relever pour plus de la moitié de l'échantillon. Cette thématique est dans bien des cas reliée aux autres défis (p. ex.: vivre de manière autonome, jugement des autres en milieu de travail ou dans les relations sociales, problèmes financiers). Une autre source d'anxiété a été relevée: l'imputabilité. Vivre sous condition était anxiogène; les participants se disaient très préoccupés par l'idée de faire une erreur qui pourrait les renvoyer en détention, même s'ils étaient déterminés à mener à bien leur réinsertion en communauté. Des sept individus ayant abordé ce sous-thème, un seul n'était pas de la clientèle provinciale.

C'est le cas pour moi, je suis fragile psychologiquement. Donc là, être imputable, c'est juste de mettre encore plus de pression que... c'est plus écrasant encore que... ça... ça le serait déjà là parce que... j’ai beaucoup beaucoup de stress là tu sais. Quand t'es incapable de gagner ta vie puis tu ne vois pas comment, un avenir prévisible [...] c'est facile de se laisser... écraser. Puis là, ce stress qu' ils rajoutent. - Félix

\section{La stigmatisation}

La stigmatisation est une thématique au cœur des difficultés de la réinsertion sociale des participants. Elle avait aussi des croisements avec les autres défis. La stigmatisation affectait les relations interpersonnelles, l'accès à l'emploi et au logement et ultimement, la motivation dans le processus de réinsertion sociale. La stigmatisation était ressentie dans la relation aux autres $(9 / 16)$, comme en témoignent les propos d'un employeur rapportés par Olivier:

[l'employeur:] «Nous autres du monde de même [délinquants sexuels], on veut rien savoir de ça, ça passe pas. Fait que tiens, on te doit 350 piasses cash, prends ça, va-t'en puis viens plus jamais icitte.» [Plus tard, le participant explique:] «Mais quand tu as cette étiquette-là, bien souvent, la perception publique, c'est que tu as rien fait de bon, puis tu feras jamais rien de bon non plus. C'est ça qu'il faut se débarrasser de, puis d'être capable de passer à d'autres choses je pense." - Olivier

Les participants ont fait part de leur peur du jugement par les autres et ont vu ces craintes confirmées, puis alimentées par toutes sortes de situations où ils ont effectivement été jugés, stigmatisés et rejetés. Plusieurs ont souligné que leur estime personnelle s'en voyait affectée. 
D'une manière bien différente, quelques participants (5/16) ont précisé se sentir stigmatisés par les conditions légales imposées et les règlements des maisons de transition qui les faisaient sentir comme s'ils avaient une «étiquette dans le front». Le fait d'être sous conditions, d'avoir à rendre des comptes et d'avoir un couvre-feu figurait parmi les explications. Pour certains délinquants sexuels, c'est l'étiquette "prédateur» qu'ils ressentaient en raison du nombre important et de la nature des conditions qu'ils avaient à respecter.

Dans le fond, d'être traité un peu comme, avoir l'étiquette dans le front «pédophile». Je te dirais que c'est une des choses que j’ai trouvé le plus difficile dans... de me sentir traité de cette façon-là. - Gérard

Des distinctions importantes ont été observées entre les profils de délinquance quant à la stigmatisation vécue. Les délinquants sexuels et plus particulièrement ceux ayant un antécédent lié à la pédophilie percevaient le stigma associé aux délits commis comme étant plus lourd à porter. Cet aspect sera développé davantage dans la discussion.

\section{L'hébergement en maison de transition}

L'encadrement constituait un défi pour la grande majorité des individus rencontrés (13/16) (ceux qui n'ont pas mentionné ce défi sont de la clientèle fédérale). Certains participants avaient du mal avec les règlements, principalement l'interdiction de fréquenter les résidents à l'extérieur et l'obligation de travailler/de se chercher un emploi, alors que ces éléments ne figurent pas dans leurs conditions de libération. Ils semblaient avoir du mal à comprendre le bien-fondé de ces règles et avaient l'impression que cela nuisait à leur cheminement. Autrement, la fréquence et la quantité des modalités de vérification exigées donnaient l'impression d'un «cadre exagéré». Quelques participants éprouvaient des difficultés dans leur relation aux intervenants, notamment en lien avec le mandat de sécurité.

Ils t'aident pas ici. Je leur ai dit l'autre fois. Je l'ai dit à mon intervenant. "Quand on comprend que votre job c'est d'être l'bélicoptère qui spote pour surveiller comment... je me... balade dans toutes mes conditions. [...] C'est juste ça votre job. C'est de vous assurer que je ne suis pas un danger pour la société malgré la longue liste de things to do que j'ai. C'est juste ça». Mon état bumain, ils s'en foutent. $A$ moins qu'il représente un danger pour la société. - André 
De plus, la cohabitation avec une trentaine d'individus ainsi que l'intégration de délinquants sexuels faisaient en sorte que les participants avaient bien peu d'intimité et qu'ils se sentaient irrités par la présence et les comportements des autres résidents.

\section{Retour sur les profils étudiés}

Alors que les particularités observées chez certains profils d'individus ont été relevées, lorsque pertinentes, à travers la description des défis de la réinsertion sociale, voici un compte-rendu des principales observations.

Des particularités ont été relevées pour les résidents sous mandat fédéral en ce qui a trait aux défis du retour à la liberté, des relations conjugales et de l'hébergement. La clientèle provinciale s'est distinguée quant aux préoccupations financières, à l'anxiété relative à l'imputabilité, aux difficultés d'accès à des programmes et à tous les défis liés à l'hébergement en maison de transition (encadrement, relations avec les intervenants, cohabitation). Finalement, des spécificités ont été observées chez les individus ayant un antécédent de nature sexuelle principalement pour la recherche d'emploi, les relations sociales (de tous types) et la stigmatisation (tous les sous-thèmes).

\section{Discussion}

L'étude avait pour objectif principal d'offrir une meilleure compréhension des défis vécus par des ex-détenus dans le processus de réinsertion sociale, objectif rendu possible en étudiant l'expérience subjective d'un échantillon de 16 résidents de maisons de transition. L'analyse des données met en lumière six catégories de défis qui affectent plusieurs sphères de la vie de ces individus. Il existe de nombreux parallèles entre ces éléments et les travaux sur la réinsertion sociale, comme: les difficultés d'insertion professionnelle, les problématiques relationnelles, l'impact du casier judiciaire et l'accès limité aux programmes (Brown, 2004; Comfort et al., 2018; Petersilia, 2003; Tan et al., 2016). Les résultats offrent également certaines contributions originales. En effet, alors que le manque de formation est reconnu comme un obstacle à la réinsertion sociale (Petersilia, 2003), aucune recherche ne s'est penchée sur les difficultés associées à un retour aux études. De plus, peu de recherches considèrent comment les interventions, dont celles offertes 
en maison de transition, peuvent elles-mêmes poser des défis pour la clientèle judiciarisée.

Les résultats lèvent le voile sur une situation complexe où certains thèmes abordés s'associent à la fois à des facteurs de réussite et à des facteurs de risques. Il a été démontré empiriquement que l'emploi est un des éléments les plus associés à la réussite de la réintégration à la société tant pour la délinquance générale (Laub et Sampson, 2003) que pour la délinquance sexuelle (Kruttschnitt, Uggen et Shelton, 2000; Laws et Ward, 2011), et qu'inversement, éprouver des difficultés dans la sphère occupationnelle est une situation augmentant le risque de récidive (Gendreau et al., 1996). La littérature met également en évidence l'apport positif des relations familiales, conjugales ou d'amitié prosociales (Laub et Sampson, 2003; Sampson et Laub, 1993; Valera, Brotzman, Wilson et Reid, 2017) et la stabilité financière (Petersilia, 2003). Autrement, des difficultés relatives à l'hébergement (Lutze, Rosky et Hamilton, 2014) et le fait de vivre avec des problématiques criminogènes (p. ex. : consommation, cognitions antisociales, dont les déviances sexuelles) (Gendreau et al., 1996) sont susceptibles d'augmenter le risque de récidive. En définitive, les défis mentionnés par les résidents sont légitimes dans l'optique où une intervention permettant de les relever devrait généralement minimiser leur risque de récidive et maximiser l'accès à des facteurs de réussite.

La relation entre les défis de la réinsertion sociale et les facteurs de réussite du processus tient également sur le plan théorique, avec les théories du désistement. Plusieurs auteurs ont établi que le désistement criminel n'est possible que si la société accepte concrètement la réintégration de l'individu; les personnes judiciarisées ne peuvent croire en leur transformation que si la communauté croit en la réinsertion sociale (Maruna, 2001, 2012) et qu'il y a un étiquetage positif (Göbbels, Ward et Willis, 2012; Maruna et al., 2004, 2009). La théorie de la transformation cognitive par Giordano et al. (2002) met à l'avant-plan le rôle de l'environnement social. L'idée au cœur de cette théorie est que la réussite de la réinsertion sociale et ultimement le désistement criminel reposent sur une interaction entre l'individu et la structure sociale; les personnes judiciarisées ont un rôle dans leur réinsertion et des opportunités dans l'environnement social (ou des grappins à changement) sont nécessaires (Giordano et al., 2002). Dans le cas de la présente étude, c'est souvent sur le plan de ces occasions dans la structure sociale que les individus se voient freinés dans leur processus de réinsertion 
sociale ou de désistement criminel. En fait, plusieurs participants ont fait part de leur ouverture au changement et de leur désir de se réinsérer et de vivre en conformité avec les lois. Toutefois, alors qu'une occasion d'emploi, d'accès à un programme ou d'établissement de liens prosociaux aurait été un élément ayant le potentiel de soutenir le cheminement de ces individus et de catalyser le changement, cette même occasion s'avérait plutôt problématique dans le parcours vers la légitimité. L'hébergement en maison de transition peut d'ailleurs être conçu comme pouvant soutenir ces individus. Or, les défis rencontrés (encadrement strict, relations difficiles avec les intervenants) font en sorte que cela ne s'avère pas nécessairement un grappin à changement (Giordano et al., 2002).

La stigmatisation des personnes judiciarisées et certaines contraintes légales sont deux éléments concourant à la réduction des opportunités disponibles dans la structure sociale. Des non-sens entre les conditions de libération et les objectifs de réinsertion sociale peuvent générer un climat de contre-productivité. Bien que la sécurité du public demeure la préoccupation principale, ces constats proposent une réflexion sur l'introduction d'une flexibilité sur le plan des conditions de libération de sorte qu'elles aient moins d'effets iatrogènes sur le succès de la réinsertion sociale. En somme, bien que certaines difficultés rencontrées dans le cadre de la réinsertion sociale reposent sur une responsabilité individuelle (p. ex.: adhérence à un mode de vie prosocial, gestion des finances personnelles), une part non négligeable tient à l'environnement légal et social dans lequel ces individus se réinsèrent.

L'une des contributions intéressantes de l'étude est son volet exploratoire des particularités associées à différents profils. Ces résultats préliminaires font part d'une expérience différenciée en fonction de certaines caractéristiques individuelles. D'une part, la longueur des peines et des périodes de transition influence la réintégration des individus. La clientèle fédérale, généralement plus âgée, a vécu une coupure plus importante avec la société; le retour à la liberté peut être plus difficile suivant l'institutionnalisation. On comptait davantage de célibataires parmi ces résidents et cela pourrait expliquer pourquoi eux, plus que les autres, devaient relever certains défis. Par exemple, les célibataires seraient plus à risque de vivre des difficultés associées au développement d'une relation intime et se trouveraient davantage dans l'obligation de chercher un nouveau logement en comparaison de leurs homologues en couple, plus enclins à retourner vivre avec leur conjointe. 
La durée plus importante des sentences pourrait être la raison de la prépondérance de célibataires dans l'échantillon fédéral, la durée de la séparation étant le principal facteur explicatif dans la relation incarcération-dissolution conjugale (Massoglia, Remster et King, 2011). Pour ce qui est de la clientèle provinciale, il semble que la courte durée de la période de transition puisse créer une pression à se réintégrer rapidement. Le stress engendré par l'idée de devoir être prêt rapidement à vivre de manière autonome pourrait expliquer la moindre tolérance aux motifs d'irritation de la vie en centre d'hébergement et que l'imputabilité ou de faibles moyens financiers soient particulièrement anxiogènes. De plus, la difficulté d'accéder aux programmes en communauté ne touchait que cette clientèle (à l'exception d'un cas) et les résidents ont précisé avoir moins d'accès à des programmes en établissement de détention provincial. Cet accès restreint aux programmes permettant de traiter des problématiques criminogènes (p. ex. : toxicomanie, déviance sexuelle, gestion des émotions) à différentes étapes du processus judiciaire pourrait également laisser ces individus dans un état de fragilité expliquant les particularités relevées.

D'autre part, les résultats laissent supposer que la nature des antécédents affecte le processus de réinsertion sociale. La stigmatisation aurait des effets plus importants pour la clientèle sexuelle. Le lourd stigmate lié aux infractions sexuelles serait fonction de la peur et de la colère générées par ce type de délit (Göbbels et al., 2012; Harris, 2014; Laws et Ward, 2011). Selon les participants, cela justifierait des jugements plus sévères pour les infractions liées à la pédophilie. La réaction face aux crimes sexuels serait influencée par l'association de ce crime à la prédation. La majorité des délinquants sexuels ne correspond toutefois pas à ce stéréotype (Laws et Ward, 2011). La stigmatisation explique en partie les spécificités relevées. Les nombreuses conditions de libération conditionnelle, notamment celles prévues à l'article 161 (p. ex.: accès restreint aux parcs/environnement où se trouvent des personnes mineures), constituent un autre élément derrière les contraintes supplémentaires que cette clientèle rencontre (voir Grossi, 2017). Enfin, certaines des difficultés vécues lors de la réinsertion sociale ne sont pas nouvelles, dans la mesure où ce profil de délinquant présente généralement des déficits (notamment sur le plan interpersonnel) qui, dans bien des cas, a mené les participants à la délinquance. 


\section{Conclusion}

Visant une compréhension approfondie des défis associés à la réinsertion sociale d'hommes hébergés en maison de transition, les résultats de la présente étude ont permis de souligner la pluralité des difficultés rencontrées et l'importance de les traiter dans une perspective de désistement du crime. Les conclusions de cette étude doivent être appréciées à la lumière du caractère exploratoire du devis de recherche utilisé. Les biais d'échantillonnage (tri expertisé, participation volontaire) et la non-saturation empirique sont les principales limites. Bien que la portée des résultats soit limitée, le devis exploratoire a permis de déterminer plusieurs pistes qui gagneraient à être étudiées davantage. C'est le cas notamment des éléments susceptibles de créer un climat de contreproductivité. De plus, l'analyse soulève que les difficultés rencontrées lors de la réinsertion sociale seraient influencées par certaines caractéristiques du parcours antérieur à la remise en liberté (type de délit commis, déficits antérieurs, durée de la peine). Dans une perspective d'intervention, l'amélioration des connaissances sur les tendances observées selon ces profils est susceptible d'être utile au développement d'une pratique adaptée aux particularités individuelles.

\section{Références}

Adams, W. C. (2015). Conducting semi-structured interviews. Dans K. E. Newcomer, H. P. Hatry et J. S. Wholey (dir.), Handbook of practical program evaluation (4e éd., p. 492-505). Hoboken, NJ: John Wiley \& Sons, Inc.

Association des services de réhabilitation sociale du Québec. (2014). Réinsertion sociale [Dossier thématique]. Repéré à http://asrsq.ca/fr/pdf/dossiers-thematiques/reinsertion-sociale.pdf

Brown, J. D. (2004). Challenges facing canadian federal offenders newly released to the community. Journal of Offender Rehabilitation, 39(1), 19-35.

Comfort, M., Krieger, K. E., Landwehr, J., McKay, T., Lindquist, C. H., Feinberg, R., ... Bir, A. (2018). Partnership after prison: Couple relationships during reentry. Journal of Offender Rebabilitation, 57(2), 188-205.

Gendreau, P., Little, T. et Goggin, C. (1996). A meta-analysis of the predictors of adult offender recidivism: What works! Criminology, 34(4), 575-608.

Giordano, P. C., Cernkovich, S. A. et Rudolph, J. L. (2002). Gender, crime, and desistance: Toward a theory of cognitive transformation. American Journal of Sociology, 107(4), 990-1064.

Göbbels, S., Ward, T. et Willis, G. M. (2012). An integrative theory of desistance from sex offending. Aggression \& Violent Behavior, 17(5), 453-463. 
Grossi, L. M. (2017). Sexual offenders, violent offenders, and community reentry: Challenges and treatment considerations. Aggression and Violent Behavior, 34, 59-67.

Gunnison, E. et Helfgott, J. B. (2011). Factors that hinder offender reentry success: A view from community corrections officers. International Journal of Offender Therapy and Comparative Criminology, 55(2), 287-304.

Hardcastle, L., Dowse, L., McGillivray, J., Newton, D., Rowe, S., Crosbie, J. et Giles, M. (2018). A qualitative study of the experiences of ex-prisoners who are seeking employment, the experiences of practitioners who work with ex-prisoners who are seeking employment and models of practice used. Sydney, Australie: UNSW Sydney.

Harding, D. J., Wyse, J. J. B., Dobson, C. et Morenoff, J. D. (2014). Making ends meet after prison. Journal of Policy Analysis and Management, 33(2), 440-470.

Harris, D. A. (2014). Desistance from sexual offending: Findings from 21 life history narratives. Journal of Interpersonal Violence, 29(9), 1554-1578.

Holzer, H. J., Raphael, S. et Stoll, M. A. (2004). How willing are employers to hire ex-offenders? Focus, 23(2), 40-43.

Kruttschnitt, C., Uggen, C. et Shelton, K. (2000). Predictors of desistance among sex offenders: The interaction of formal and informal social controls. Justice Quarterly, 17(1), 61-87.

Laub, J. H. et Sampson, R. J. (2003). Shared beginnings, divergent lives. Harvard University Press.

Laws, D. R. et Ward, T. (2011). Desistance from sex offending: Alternatives to throwing away the keys. New York, NY: Guilford Press.

Lutze, F. E., Rosky, J. W. et Hamilton, Z. K. (2014). Homelessness and reentry: A multisite outcome evaluation of Washington state's reentry housing program for high risk offenders. Criminal Justice and Behavior, 41(4), 471-491.

Maruna, S. (2001). Making good: How ex-convicts reform and rebuild their lives. Washington, DC: American Psychological Association.

Maruna, S. (2012). Elements of successful desistance signaling. Criminology $\mathfrak{\sigma}$ Public Policy, 11(1), 73-86.

Maruna, S., Lebel, T. P., Mitchell, N. et Naples, M. (2004). Pygmalion in the reintegration process: Desistance from crime through the looking glass. Psychology, Crime of Law, 10(3), 271-281.

Maruna, S., Lebel, T. P., Naples, M. et Mitchell, N. (2009). Looking-glass identity transformation: Pygmalion and Golem in the rehabilitation process. Dans B. M. Veysey, J. Christian et D. J. Martinez (dir.), How offenders transform their lives (p. 30-51). Cullompton, Royaume-Uni: Willan Publishing.

Massoglia, M., Remster, B. et King, R. D. (2011). Stigma or separation? Understanding the incarceration-divorce relationship. Social Forces, 90(1), 133-155.

Miles, M. B., Huberman, A. M. et Saldaña, J. (2014). Fundamentals of qualitative data analysis. Dans M. B. Miles, A. M. Huberman et J. Saldaña (dir.), Qualitative data analysis: A methods sourcebook ( $3^{\mathrm{e}}$ éd., p. 69-104). Thousand Oaks. 
Moore, K. E., Stuewig, J. B. et Tangney, J. P. (2016). The Effect of stigma on criminal offenders' functioning: A longitudinal mediational model. Deviant Behavior, 37(2), 196-218.

Morani, N. M., Wikoff, N., Linhorst, D. M. et Bratton, S. (2011). A description of the self-identified needs, service expenditures, and social outcomes of participants of a prisoner-reentry program. The Prison Journal, 91(3), 347-365.

Nhan, J., Bowen, K. et Polzer, K. (2017). The reentry labyrinth: The anatomy of a reentry services network. Journal of Offender Rebabilitation, 56(1), 1-19.

Petersilia, J. (2003). When prisoners come home: Parole and prisoner reentry. New York, NY: Oxford University Press.

Roulston, K. (2014). Analysing interviews. Dans U. Flick (dir.), The SAGE bandbook of qualitative data analysis (p. 297312). Londres, Royaume-Uni: SAGE Publications Ltd.

Rydberg, J. (2018). Employment and housing challenges experienced by sex offenders during reentry on parole. Corrections, 3(1), 1537.

Sampson, R. J. et Laub, J. H. (1993). Crime in the making: Pathways and turning points through life. Harvard University Press.

Seiter, R. P. et Kadela, K. R. (2003). Prisoner reentry: What Works, what does not, and what is promising. NCCD News, 49(3), 360-388.

Tan, X. X., Chu, C. M. et Tan, G. (2016). Factors contributing towards stigmatisation of offenders in Singapore. Psychiatry, Psychology $\mathfrak{F}$ Law, 23(6), 956-969.

Thornton, D. G. (2018). The effects of institutional discrimination on the successful reentry of ex-offenders (Mémoire de maîtrise, Université de Houston). Repéré à https://uhcl-ir.tdl.org/handle/10657.1/1415

Trahan, A. et Stewart, D. M. (2013). Toward a pragmatic framework for mixedmethods research in criminal justice and criminology. Applied Psychology in Criminal Justice, 9(1), 59-74.

Valera, P., Brotzman, L., Wilson, W. et Reid, A. (2017). "It's hard to reenter when you've been locked out": Keys to offender reintegration. Journal of Offender Rehabilitation, 56(6), 412-431.

Visher, C., LaVigne, N. et Travis, J. (2004). Returning home: Understanding the challenges of prisoner reentry. Urban Institute Justice Policy Center. Repéré à http://webarchive.urban.org/UploadedPDF/410974_ReturningHome_ MD.pdf

\section{Challenges to reintegration: Barriers to reentry encountered by ex-convicts in halfway houses}

ABSTRACT - Reintegration is a difficult process and individuals who have been affected by the justice system can benefit from interventions that help them to be autonomous, law-abiding, and integrated into their communities. Current research, however, seldom goes beyond identifying the difficulties in this process. Our qualitative study was an in-depth exploration of the challenges encountered by ex-convicts who had been pro- 
vided with lodging in halfway houses as part of their reentry process. Six categories of challenges were identified following a content analysis of the interviews $(n=16)$ : return to society, financial and occupational situation, social network, personal development, stigmatization, and living in halfway houses. Experiences specific to certain profiles (length of sentence, sexual delinquency) are noted. Results are discussed in relation to desistance theories. Among other things, the study highlights the ways in which legal conditions and the stigmatization of offenders can lead to counter-productive outcomes.

KEYWORDS - Reentry, parole, intervention, semi-structured interviews, sex offenders.

\section{Los desafíos de la reinserción social. Una mirada iluminada sobre la realidad vivida por la clientela en casa de transición}

RESUMEN - La reinserción social es un proceso plagado de trampas a través del cual un individuo que enfrenta un proceso judicial puede beneficiar de intervenciones que le permiten ser una persona autónoma, integrada a su comunidad y respetuosa de las leyes. Como generalmente el estado actual del conocimiento no permite ir más allá de la identificación de las dificultades que ocurren en este proceso, este estudio cualitativo exploratorio tiene como objetivo estudiar a profundidad los desafios encontrados por algunos hombres en los centros de reinserción social, en el marco de sus procesos de reinserción a la sociedad. El análisis de contenido de las entrevistas $(n=16)$ permitió diferenciar seis categorías de desafios, ya sean el retorno a la libertad, la situación financiera y ocupacional, la red social, el desarrollo personal, la estigmatización, y el alojamiento en una casa de transición. Particularidades de algunos perfiles (duración de la sentencia, delincuencia sexual) son puestos en relieve. Los resultados son discutidos a la luz de las teorías del desistimiento delictivo. Entre otras cosas, este ejercicio subraya que algunas condiciones legales y la estigmatización de las personas judicializadas crean desafíos contra productivos.

PALABRAS CLAVE - Reinserción social, intervenciones, liberación condicional, entrevistas semi-directivas, delincuencia sexual. 